\title{
DECOMPOSIÇÃO DE LODO DE ESGOTO E COMPOSTO DE LODO DE ESGOTO EM NITOSSOLO HÁPLICO
}

\author{
Sarah Mello Leite Moretti ${ }^{(1)}$, Edna Ivani Bertoncini ${ }^{(2) *}$ e Cassio Hamilton Abreu Junior ${ }^{(3)}$ \\ (1) Universidade de São Paulo, Centro de Energia Nuclear na Agricultura, Programa de Pós-graduação em Ciências, Piracicaba, São \\ Paulo, Brasil. \\ (2) Agência Paulista de Tecnologia dos Agronegócios, Polo Centro Sul; Piracicaba, São Paulo, Brasil. \\ (3) Universidade de São Paulo, Centro de Energia Nuclear na Agricultura, Piracicaba, São Paulo, Brasil. \\ * Autor correspondente. \\ E-mail: ebertoncini@apta.sp.gov.br
}

\section{RESUMO}

O uso agrícola de lodo de esgoto e derivados no Estado de São Paulo é regulamentado por norma federal e por norma paulista, que recomendam que esses materiais quando incorporados a solos agrícolas devem apresentar no mínimo 30 \% de degradação do carbono total. Para observar as frações de degradação de um lodo de esgoto e de um composto, produzido a partir do mesmo lodo juntamente com poda de árvore urbana triturada, realizaram-se dois ensaios de biodegradação de $\mathrm{C}$. As doses dos resíduos foram adicionadas em frascos contendo $500 \mathrm{~g}$ de solo coletado na profundidade de 0,00-0,20 m de um Nitossolo Háplico Álico textura argilosa. No ensaio I, as doses de lodo (L) e composto (C) utilizadas consideraram percentuais da necessidade em $\mathrm{N}$ para a cultura da cana-de-açúcar, e expressas em $\mathrm{Mg} \mathrm{ha}^{-1}$ de resíduo (base úmida): L1 21,2 (100 \%); L2 42,4 (200 \%); C1 69,4 (50 \%); C2 138,9 (100 \%); e C3 277,8 (200 \%). O ensaio II foi realizado apenas com o lodo de esgoto, utilizando-se doses $5,10,15$ e 20 vezes maiores que aquelas recomendadas no ensaio I: L3 120; L4 240; L5 360; e L6 480, simulando sucessivas aplicações do resíduo no solo. $\mathrm{O} \mathrm{C}-\mathrm{CO}_{2}$ liberado foi quantificado por meio da medida de condutividade elétrica. O lodo de esgoto no ensaio I apresentou menores frações de degradação quando comparado ao composto orgânico, mas a taxa de decomposição do composto foi menor, provavelmente pela presença de substâncias recalcitrantes resultantes do processo de humificação. A fração de degradação dos resíduos no solo atingiu valor próximo a $30 \%$ apenas para a dose de lodo de esgoto 20 vezes superior à dose exigida pela cultura, e assim não poderiam ser utilizados em solos agrícolas argilosos. Observou-se a necessidade de revisão desse valor adotado pela legislação paulista de uso de resíduos orgânicos em solos.

Palavras-chave: lodo sanitário, compostagem, respirometria. 


\title{
ABSTRACT: DECOMPOSITION OF SEWAGE SLUDGE AND SEWAGE SLUDGE COMPOST IN A HAPLUDOX SOIL
}

\begin{abstract}
The agricultural use of sewage sludge and derivatives is to be carried out according to requirements established by Brazilian federal legislation and by legislation of the State of São Paulo, which requires that these organic materials must have at least $30 \%$ of the total organic carbon degraded when incorporated in agricultural soils. To observe the degradation rates of sewage sludge and of organic compost produced from the same sewage sludge mixed with ground material from tree pruning, we performed two respirometry tests according to the modified method of Cetesb (1999). The waste materials were added to bottles containing $500 \mathrm{~g}$ of soil collected from the 0.00-0.20 m layer of a Nitossolo Háplico Álico (Hapludox) soil. In Test $I$, for sewage sludge $(L)$ and sewage sludge compost $(C)$, application rates were calculated to provide percentages of $\mathrm{N}$ requirements for one sugarcane cycle, in $\mathrm{Mg} \mathrm{ha}^{-1}$ (wet basis) of waste: L1 21.2 (100\%); L2 42.4 (200\%); C1 69.4 (50\%); C2 138.9 (100\%); and C3 277.8 (200\%). Test II was conducted only with sewage sludge, at the rates of 5, 10, 15, and 20 times higher than those recommended in Test I: L3 120; L4 240; L5 360; and L6 480. The $\mathrm{CO}_{2}$-C released was quantified by measuring electrical conductivity. In Test I, the treatments with sewage sludge had lower degradation rates than treatments with sewage sludge compost, probably due to the presence of recalcitrant substances formed during the humification phase of the composting process. The $30 \%$ degradation rate was attained only by the application rate that was 20 times higher than the rate recommended for a cycle of sugarcane, and thus these waste products could not be used in clayey agricultural soils. Therefore, it is necessary to review the values established by legislation in the State of São Paulo.
\end{abstract}

Keywords: biossolid, composting, respirometry assay.

\section{INTRODUÇÃO}

O lodo de esgoto é gerado em grandes quantidades, e o volume produzido tende a aumentar nos próximos anos. De acordo com as diretrizes da Política Nacional de Resíduos Sólidos (PNRS), a disposição do lodo de esgoto em aterros sanitários será restringida, pois há melhores alternativas de destino possíveis para esse resíduo orgânico, como o reaproveitamento agrícola (fertilizante orgânico) (Wang et al., 2008) e, também, o reaproveitamento industrial (fabricação de cerâmicas e tijolos) (Andreoli, 2006). A mesma Lei prevê melhorias no saneamento básico, como o aumento das estações de tratamento de esgoto no Brasil; logo, sua geração será cada vez maior nos próximos anos (Brasil, 2010).

Assim, alternativas de destino da grande quantidade de material orgânico gerado devem ser estudadas. $\mathrm{O}$ uso agrícola do lodo de esgoto pode ser considerado alternativa de destino, uma vez que em razão do alto teor de matéria orgânica e da presença de alguns nutrientes como $\mathrm{N}, \mathrm{P}$ e micronutrientes (Bertoncini et al., 2004), seu uso agrícola pode proporcionar melhorias ao solo e aumento na produtividade agrícola (Bovi et al., 2007; Melo et al., 2007; Wang et al., 2008).

Aumento na produtividade de colmos de cana-de-açúcar com o uso de diferentes doses de lodo de esgoto combinadas ou não com o uso de fertilizantes foi observado por Franco et al. (2010). Neste estudo, também foi possível observar, no ano seguinte, efeito residual da aplicação anterior de lodo de esgoto, tanto sobre a produção de colmos como a produção de açúcar. Segundo os autores, esse efeito foi devido a maior mineralização da matéria orgânica, no segundo ano de cultivo. Aumento na produtividade de grãos de soja foi observado por Motaghian e Bahmanyar (2010), em estudo realizado com uso de $40 \mathrm{Mg} \mathrm{ha}^{-1}$, assim como aumento no diâmetro do caule das plantas de soja.

O lodo de esgoto produzido na estação de tratamento de esgoto da cidade de Franca, São Paulo, Brasil (ETE-Sabesp/Franca), foi pioneiramente utilizado na cultura do café, desde 1999. Porém, a partir de 2006, o uso de lodos sanitários foi restringido pela resolução Conama № 375 (Brasil, 2006a), que especifica os limites máximos permitidos de metais pesados e patógenos, além de proibir o seu uso em hortaliças, raízes e tubérculos, como medida preventiva de saúde pública.

Portanto, a maioria dos lodos de esgotos produzidos no Brasil não se enquadra na categoria passível de uso agrícola, necessitando de pós-tratamento para tal uso, sendo o processo de compostagem de lodos sanitários indicado para redução de vetores, de acordo com a Resolução Conama No 380 (Brasil, 2006b). Com o processo de compostagem, também, é possível obter um material orgânico com melhores características físico-químicas, químicas e microbiológicas para uso agrícola sustentável do resíduo (Corrêa et al., 2006; Hua et al., 2009; Khalil et al., 2011).

Apesar de todos os benefícios já conhecidos da fertilização orgânica, suas características e seu manejo inadequado podem proporcionar desequilíbrios em solos. Os microrganismos 
decompositores da matéria orgânica necessitam de 30 partes de $\mathrm{C}$ para uma parte de $\mathrm{N}$, em que 20 partes serão perdidas no processo via $\mathrm{CO}_{2}$ e 10 partes irão compor a estrutura desses microrganismos (Pereira Neto, 1989). Dessa forma, a relação C/N de determinado material orgânico adicionado ao solo irá influenciar a cinética de sua degradação pelos microrganismos (Aita, 1997). Materiais orgânicos adicionados ao solo com relação $\mathrm{C} / \mathrm{N}$ maior do que 30/1 tendem a favorecer o processo de imobilização de $\mathrm{N}$, uma vez que haverá um déficit de $\mathrm{N}$ necessário para sua decomposição e assimilação pelos microrganismos decompositores. $\mathrm{O} \mathrm{N}$ necessário para compor a estrutura proteica desses microrganismos será retirado da matéria orgânica nativa do solo (MOS), podendo ocasionar deficiência de $\mathrm{N}$ às plantas cultivadas (Victoria et al., 1992; Kiehl, 1985).

Por sua vez, materiais orgânicos adicionados ao solo com relação $\mathrm{C} / \mathrm{N}$ baixa, menor que 15:1, tendem a mineralizar o $\mathrm{N}$ e imobilizar o $\mathrm{C}$ nativo do solo, podendo ocasionar depleção no teor de MOS (Fontaine et al., 2003), fato não desejável para fertilidade de solos sob condições tropicais, geralmente pobres em matéria orgânica. Este fenômeno denomidado de "efeito priming" foi observado em estudo realizado por Hernandez et al. (1988) em que maiores teores de $\mathrm{C}$ foram observados em solos que nunca receberam resíduos orgânicos quando comparados aos solos com uso de resíduos orgânicos.

Em razão disso, a Companhia de Tecnologia de Saneamento Ambiental (Cetesb) definiu, para o Estado de São Paulo, um valor mínimo de 30 \% de degradação do C-orgânico de lodos biológicos, a fim de evitar o acúmulo de material orgânico recalcitrante no solo (Cetesb, 1999). Contudo, cabe ressaltar que há resíduos que podem apresentar frações de degradação do C-orgânico maior que $100 \%$, representando a decomposição, inclusive, da MOS, condição não desejada em solos sob condições tropicais, pobres em matéria orgânica, e essa condição não foi observada nessa legislação. A adição ao solo de um material constituído por componentes orgânicos mais complexos e insolúveis pode favorecer a degradação da MOS por microrganismos especializados (Fontaine et al., 2003). Os lodos biológicos gerados podem ocasionar tal fenômeno, uma vez que durante o processo de tratamento do esgoto há a degradação dos compostos orgânicos lábeis.

Redução no teor de $\mathrm{C}$ do solo após aplicação de 10 , 20 , e $40 \mathrm{Mg} \mathrm{ha}^{-1}$ de um lodo de esgoto foi observado por Guedes et al. (2006), confirmando os resultados obtidos por Hsieh et al. (1981) que avaliaram a decomposição de lodos de esgoto em condições de laboratório por meio da medida da evolução de $\mathrm{CO}_{2}$. Também foram observadas reduções nos teores de MOS, em estudo realizado por Firmino et al. (2015) e Medeiros (2012) com uso de diferentes lâminas de água residuária na irrigação da cultura do pinhão manso. Esses autores obtiveram reduções de 57,9 e $12,3 \%$ nos teores de MOS, após o terceiro e segundo cultivo, respectivamente.

As frações de degradação encontradas para lodos de esgoto adicionados ao solo estão na faixa de 20 a $60 \%$ do C orgânico total (Andrade et al., 2006a); para compostos orgânicos, esses valores tendem a ser menores que $20 \%$, em razão da presença de material orgânico de difícil decomposição, resultantes do processo de humificação. Dessa forma, é importante conhecer as características do material orgânico a ser utilizado, bem como a natureza dos compostos que o constituem, a fim de evitar danos e proporcionar melhorias à fertilidade do solo.

Sabendo-se da importância do uso agrícola do lodo de esgoto e composto de lodo de esgoto e que as frações de degradação desses materiais podem variar em função de fatores como a dose aplicada e as características físico-químicas do material orgânico, objetivou-se por meio deste estudo avaliar as taxas de decomposição de um lodo de esgoto anaeróbio e um composto produzido a partir do mesmo lodo e palha de árvore urbana, triturada, em um solo argiloso, de modo a prever seu comportamento e a liberação de nutrientes para o manejo agrícola.

\section{MATERIAL E MÉTODOS}

O lodo de esgoto utilizado no ensaio de biodegradação de C foi produzido pela ETE-Franca, Sabesp, e coletado no início da montagem das pilhas de compostagem, sendo proveniente de tratamento de esgoto doméstico por processos aeróbio, anaeróbio e deságue em filtro-prensa com uso de polímeros. O composto de lodo de esgoto utilizado no ensaio de biodegradação foi proveniente do processo de compostagem do lodo de esgoto gerado nessa ETE com poda de árvore urbana triturada, partindo da relação C:N da mistura de $30: 1$, conduzido e monitorado pelo período de 120 dias, das pilhas com monitoramento de temperatura e revolvimento mecânico periódico. Os materiais utilizados nesse ensaio foram previamente caracterizados quanto às características químicas e físico-químicas, de acordo com Andrade e Abreu (2006) (Quadro 1).

$\mathrm{O}$ ensaio de biodegradação de $\mathrm{C}$ foi realizado de acordo com o método respirométrico de Bartha (Cetesb, 1999) modificado, que se baseia na captura do dióxido de carbono $\left(\mathrm{CO}_{2}\right)$ liberado pela atividade microbiana durante processo de decomposição do material orgânico presente no resíduo, em solução padronizada de hidróxido de sódio $(\mathrm{NaOH})$.

Foram conduzidos dois ensaios de degradação de C, com doses diversas dos resíduos. No ensaio I, as doses foram calculadas para fornecer porcentagens 
Quadro 1. Caracterização química do lodo de esgoto e composto de lodo de esgoto

\begin{tabular}{|c|c|c|c|c|c|c|c|c|c|c|c|c|c|c|}
\hline $\mathrm{pH}\left(\mathrm{CaCl}_{2}\right)^{(1)}$ & $\mathbf{C E}^{(2)}$ & $\mathrm{U}^{(3)}$ & $\mathrm{MO}^{(4)}$ & $\mathrm{CTC} / \mathrm{C}^{(5)}$ & $\mathbf{C}$ & $\mathbf{N}$ & $\mathbf{P}$ & $\mathbf{K}$ & $\mathbf{C a}$ & $\mathrm{Mg}$ & $\mathbf{S}$ & $\mathrm{Cu}$ & Mn & $\mathrm{Zn}$ \\
\hline & $\mathrm{mS} \mathrm{cm}^{-1}$ & \multicolumn{2}{|c|}{$\%$} & & \multicolumn{7}{|c|}{$\mathrm{g} \mathrm{kg}^{-1}$} & \multicolumn{3}{|c|}{$\mathrm{mg} \mathrm{kg}^{-1}$} \\
\hline \multicolumn{15}{|c|}{ Lodo de esgoto } \\
\hline 7,8 & 1.887 & 70 & 10 & 12 & 140 & 35 & 11 & 1 & 14 & 3 & 9 & 202 & 387 & 690 \\
\hline \multicolumn{15}{|c|}{ Composto de lodo de esgoto } \\
\hline 6,5 & 782 & 58 & 18 & 21 & 190 & 18 & 12 & 3 & 21 & 3 & 5 & 152 & 290 & 517 \\
\hline
\end{tabular}

(1) $\mathrm{pH}$ determinado em solução de $\mathrm{CaCl}_{2} 0,01 \mathrm{~mol} \mathrm{~L}^{-1} .^{(2)} \mathrm{CE}$ : Condutividade elétrica determinada em água. ${ }^{(3)}$ Teor de água a $65{ }^{\circ} \mathrm{C}$.

(4) Teor de matéria orgânica após incineração a $550{ }^{\circ} \mathrm{C}$. ${ }^{(5)} \mathrm{CTC} / \mathrm{C}$ : relação carbono na capacidade de troca catiônica do solo.

da necessidade em $\mathrm{N}$ de $100 \mathrm{~kg} \mathrm{ha}^{-1}$ para a cultura da cana-de-açúcar, considerando para isso as frações 20 e $10 \%$ de mineralização do N-total estipulado pela Resolução Conama No 375 (Brasil, 2006a) para lodo de esgoto e composto orgânico, respectivamente. Os teores de água do lodo de esgoto e composto orgânico foram de 73 e $60 \%$, respectivamente. As doses para o lodo de esgoto, em $\mathrm{Mg} \mathrm{ha}^{-1}$ (base úmida), foram: L1 21,2 ; e L2 42,4, correspondentes a 100 e $200 \%$ de $\mathrm{N}$ requerido pela cultura da cana, respectivamente; e para o composto orgânico: C1 69,4; C2 138,9; e C3 277,8, correspondentes a 50, 100 e $200 \%$ de N requerido pela cultura da cana, respectivamente.

No ensaio II, foram testadas as doses apenas de lodo de esgoto equivalentes a: L3 120; L4 240; L5 360; e L6 $480 \mathrm{Mg} \mathrm{ha}^{-1}$; doses 5, 10, 15 e 20 vezes maiores que aquela recomendada para uso agrícola do lodo de esgoto para a cultura da cana, simulando sucessivas aplicações do resíduo in natura no solo.

Os dois ensaios foram conduzidos com três repetições, totalizando 30 frascos. Cada frasco foi preparado com uma mistura de $500 \mathrm{~g}$ de solo coletado de área de Nitossolo Háplico Álico, textura argilosa, de área experimental situada na cidade de Piracicaba, SP ( $22^{\circ} 43^{\prime} 31^{\prime \prime} \mathrm{S}, 47^{\circ} 38^{\prime} 57^{\prime \prime} \mathrm{O}$ ), na profundidade de $0,00-0,20 \mathrm{~m}$, cuja caracterização química apresentou: $\mathrm{pH}\left(\mathrm{CaCl}_{2} 0,01 \mathrm{~mol} \mathrm{~L}^{-1}\right)$ 4,0; matéria orgânica $22 \mathrm{~g} \mathrm{dm}^{-3} ; \mathrm{P} 8,0 \mathrm{mg} \mathrm{dm}^{-3} ; \mathrm{K}^{+} 2,4 \mathrm{mmol}_{\mathrm{c}} \mathrm{dm}^{-3}$; $\mathrm{Ca}^{2+} 10,0 \mathrm{mmol}_{\mathrm{c}} \mathrm{dm}^{-3} ; \mathrm{Mg}^{2+} 5,0 \mathrm{mmol}_{\mathrm{c}} \mathrm{dm}^{-3}$; $\mathrm{Al}^{3+}$ 19,0 mmol $\mathrm{dm}^{-3} ; \mathrm{H}+\mathrm{Al} \mathrm{72,0} \mathrm{mmol}_{\mathrm{c}} \mathrm{dm}^{-3} ;$ soma de bases $17,4 \mathrm{mmol}_{\mathrm{c}} \mathrm{dm}^{-3} ; \mathrm{CTC}_{\mathrm{pH} 7,0} 89,4 \mathrm{mmol}_{\mathrm{c}} \mathrm{dm}^{-3} ; \mathrm{V}$ 19,0\%; B $0,3 \mathrm{mg} \mathrm{dm}^{-3}$; Cu 1,0 $\mathrm{mg} \mathrm{dm}^{-3} ; \mathrm{Fe} 37,0 \mathrm{mg} \mathrm{dm}^{-3}$; Mn 17,0 mg dm${ }^{-3}$; Zn 0,6 $\mathrm{mg} \mathrm{dm}^{-3}$; e Cu 1,0 $\mathrm{mg} \mathrm{dm}^{-3}$. Adicionaram-se as doses de resíduos ao solo, e esse foi irrigado até atingir $70 \%$ da sua capacidade de campo.

Aos frascos contendo o solo tratado com os resíduos, foram adicionados potes com $50 \mathrm{~mL}$ de solução de $\mathrm{NaOH} 0,5 \mathrm{~mol} \mathrm{~L}^{-1}$, e esses foram incubados a $25 \pm 2{ }^{\circ} \mathrm{C}$, até paralisação total da produção de $\mathrm{CO}_{2}$ em três determinações consecutivas; ou seja, cada ensaio foi finalizado após as leituras da condutividade elétrica da solução de $\mathrm{NaOH} 0,5 \mathrm{~mol} \mathrm{~L}^{-1}$ apresentarem valores iguais em três determinações consecutivas. Diariamente, foi quantificado o $\mathrm{CO}_{2}$ liberado por meio de medida da condutividade elétrica da solução de $\mathrm{NaOH}$ e calculado como proposto por Rodella e Saboya (1999).

Os resultados de C- $\mathrm{CO}_{2}$ liberado foram ajustados em razão do tempo de incubação, usando equação de cinética química de primeira ordem (Latham, 1994) para obter os parâmetros indicativos de taxa de degradação da fração orgânica dos resíduos, a quantidade potencialmente degradada e o tempo de meia vida dos resíduos orgânicos (Equação 1). Os resultados ajustados foram submetidos à análise estatística pelo teste de Tukey a $5 \%$, utilizando o programa de análise estatística $R$ (versão 2.15.1).

C-degradado $=C_{0} \times\left(1-e^{-k t}\right) \quad$ Eq. 1

em que, $C$-degradado é a quantidade de $\mathrm{C}\left(\mathrm{mg} \mathrm{kg}^{-1}\right)$ liberada na forma de $\mathrm{CO}_{2}$ no tempo de realização do ensaio; $C_{0}$ é o $\mathrm{C}$ potencialmente mineralizável no tempo de realização do ensaio; $k$ é a constante de taxa de reação de degradação do $\mathrm{C}$ orgânico $\left(\mathrm{d}^{-1}\right)$; e $t$, o tempo (em dias).

Por meio dessa equação, é possível estimar a quantidade de $\mathrm{C}$ liberado na forma de $\mathrm{CO}_{2}$ (C-degradado), proveniente da atividade de degradação do material orgânico presente em cada tratamento.

A equação também estima os valores de $\mathrm{C}$ potencialmente degradado na forma de $\mathrm{CO}_{2}\left(C_{0}\right)$, que seria a quantidade potencialmente liberada de $\mathrm{C}-\mathrm{CO}_{2}$ proveniente da degradação do material orgânico, bem como a taxa de degradação $(k)$, que seria a taxa do processo de degradação do material orgânico presente em cada tratamento.

E a partir desses valores determinados e previstos pela equação, é possível também se considerar o parâmetro tempo de meia vida $\left(T_{1 / 2}\right)$, que seria o tempo, em dias, necessário para que $50 \%$ do valor estimado em $C_{0}$ fossem liberados, proveniente do processo de degradação do material orgânico de cada tratamento.

\section{RESULTADOS E DISCUSSÃO}

A estabilização das leituras no ensaio I de biodegradação, para o lodo de esgoto, ocorreu 
aos 213 dias (Figura 1). A evolução de $\mathrm{CO}_{2}$ foi crescente de acordo com as doses aplicadas, e a máxima fração de decomposição foi de 10,2\% do C-orgânico adicionado, para a dose máxima aplicada, de $200 \%\left(42,4 \mathrm{Mg} \mathrm{ha}^{-1}\right)$ de lodo de esgoto (Quadro 2). Esse resultado foi semelhante àquele observado por Boeira e Ligo (2007), em ensaio realizado com dois lodos de esgoto digeridos anaerobicamente.

No ensaio II, a mesma tendência foi observada, isto é, as frações de decomposição do lodo de esgoto se elevaram com o aumento das doses aplicadas, e a decomposição se estabilizou aos 135 dias (Figura 2); a maior fração de degradação obtida foi de $25,3 \%$, para a dose de $480 \mathrm{Mg} \mathrm{ha}^{-1}$, maior dose aplicada (Quadro 3). Cabe ressaltar que essa dose, cujo valor esteve mais próximo àquele exigido pela legislação paulista, é 20 vezes maior que a dose recomendada para a cultura da cana-de-açúcar e provavelmente conduziria a excesso de $\mathrm{N}$ na solução do solo, passível de perdas por lixiviação.

Frações de decomposição de lodo de esgoto em solos, na faixa de 20 a 60 \% do C orgânico total adicionado, têm sido relatadas (Andrade et al., 2006a, 2013). As baixas frações de degradação de lodos de esgotos em solos, quando comparado a outros resíduos orgânicos, devem-se ao fato de o material ser tratado de modos aeróbio

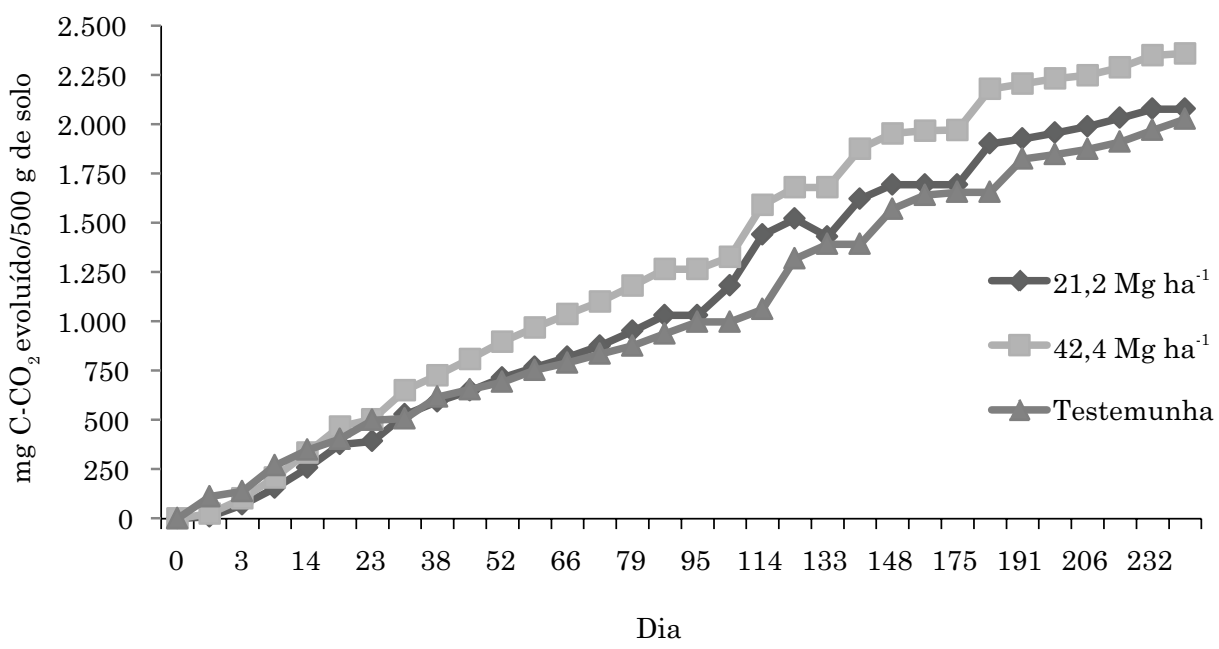

Figura 1. Evolução de $\mathrm{C}-\mathrm{CO}_{2}$ em razão das doses de lodo de esgoto aplicadas no ensaio I em Nitolosso Háplico Álico textura argilosa, coletado da camada de 0,00-0,20 m, de área localizada em Piracicaba, SP.

Quadro 2. Parâmetros da equação de cinética de primeira ordem ajustados aos dados de liberação de $\mathrm{CO}_{2}$ dos resíduos observados no ensaio I e fração de degradação, em ensaio realizado em laboratório

\begin{tabular}{|c|c|c|c|c|c|c|}
\hline Dose do resíduo & C-degradado ${ }^{(1)}$ & $\mathbf{C}_{0}{ }^{(2)}$ & $\mathrm{R}^{2(3)}$ & $\mathbf{k}^{(4)}$ & $\mathrm{T}_{1 / 2}^{(5)}$ & Fração de degradação \\
\hline \multirow[t]{2}{*}{$\mathrm{Mg} \mathrm{ha}^{-1}$} & \multicolumn{2}{|c|}{$\mathrm{mg} \mathrm{kg}^{-1}$} & & $\mathrm{~d}^{-1}$ & $\mathrm{~d}$ & $\%$ \\
\hline & \multicolumn{6}{|c|}{ Lodo de esgoto } \\
\hline 0 & $3.963,6 \mathrm{Ba}$ & $5.815,5 \mathrm{Aa}$ & 0,98 & $0,005 \mathrm{Ca}$ & $151,9 \mathrm{Aa}$ & $8,9 \mathrm{Ba}$ \\
\hline $21,2(\mathrm{~L} 1)$ & $4.202,7 \mathrm{Bb}$ & $5.483,7 \mathrm{Ab}$ & 0,98 & $0,008 \mathrm{Ba}$ & $88,9 \mathrm{Bb}$ & $9,1 \mathrm{Bb}$ \\
\hline \multirow[t]{2}{*}{42,4 (L2) } & $4.827,4 \mathrm{Ab}$ & $5.520,7 \mathrm{Ab}$ & 0,98 & $0,010 \mathrm{Aa}$ & $72,1 \mathrm{Bb}$ & $10,2 \mathrm{Ab}$ \\
\hline & \multicolumn{6}{|c|}{ Composto de lodo de esgoto } \\
\hline 0 & $3.963,6 \mathrm{Da}$ & $5.815,5 \mathrm{Da}$ & 0,98 & $0,005 \mathrm{Ba}$ & $151,9 \mathrm{Aa}$ & $8,9 \mathrm{Ba}$ \\
\hline $69,4(\mathrm{C} 1)$ & $6.164,7 \mathrm{C}$ & $8.380,0 \mathrm{C}$ & 0,99 & $0,006 \mathrm{~A}$ & $115,6 \mathrm{~B}$ & $11,3 \mathrm{~A}$ \\
\hline $138,9(\mathrm{C} 2)$ & $8.253,6 \mathrm{Ba}$ & $11.725,45 \mathrm{Ba}$ & 0,99 & 0,005 Bb & $128,6 \mathrm{ABa}$ & $11,6 \mathrm{Aa}$ \\
\hline $277,8(\mathrm{C} 3)$ & $10.297,5 \mathrm{Aa}$ & $15.827,5 \mathrm{Aa}$ & 0,99 & $0,005 \mathrm{Bb}$ & $150,2 \mathrm{Aa}$ & $12,3 \mathrm{Aa}$ \\
\hline CV (\%) & 4,0 & 6,5 & & 6,0 & 7.4 & 4,3 \\
\hline
\end{tabular}

(1) C-degradado: quantidade de carbono liberado acumulado na forma de $\mathrm{CO}_{2}$ até os 241 dias; ${ }^{(2)} \mathrm{C}_{0}$ : carbono potencialmente mineralizável em 241 dias de incubação; ${ }^{(3)} \mathrm{R}^{2}$ : coeficiente de correlação do modelo de Stanford e Smith (1972); ${ }^{(4)} \mathrm{k} \mathrm{d}{ }^{-1}$ : taxa de decomposição por dia; ${ }^{(5)} \mathrm{T}_{1 / 2}$ : tempo de meia-vida. Médias seguidas pelas mesmas letras minúsculas, entre as mesmas doses de lodo e compostos, não diferem entre si (Tukey a $5 \%$ ). Médias seguidas pelas mesmas letras maiúsculas para as doses dentro de cada resíduo não diferem entre si (Tukey a $5 \%$ ). 


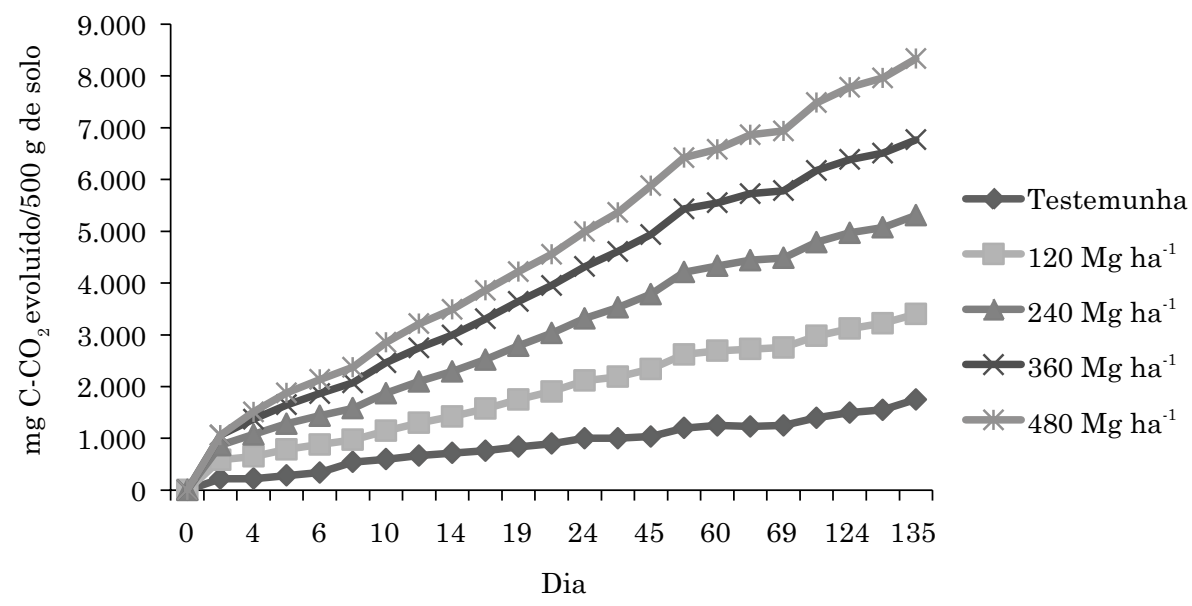

Figura 2. Evolução de $\mathrm{C}-\mathrm{CO}_{2}$ em razão das doses de lodo de esgoto aplicadas no ensaio II em Nitolosso Háplico Álico textura argilosa, coletado da camada de 0,00-0,20 m, de área localizada em Piracicaba, SP.

Quadro 3. Parâmetros da equação de cinética de primeira ordem ajustados aos dados de liberação de $\mathrm{CO}_{2}$ do tratamento com lodo de esgoto no ensaio II e fração de degradação, em ensaio realizado em laboratório

\begin{tabular}{|c|c|c|c|c|c|c|}
\hline Dose do resíduo & C-degradado $^{(1)}$ & $\mathrm{C}_{0}{ }^{(2)}$ & $\mathbf{R}^{2(3)}$ & $\mathbf{k}^{(4)}$ & $T_{1 / 2}^{(5)}$ & Fração de degradação \\
\hline $\mathrm{Mg} \mathrm{ha}^{-1}$ & \multicolumn{2}{|c|}{$\mathrm{mg} \mathrm{kg}^{-1}$} & & $\mathrm{~d}^{-1}$ & $\mathrm{~d}$ & $\%$ \\
\hline 0 & $3796,0 \mathrm{E}$ & $3796,0 \mathrm{E}$ & 0,92 & $0,042 \mathrm{BC}$ & $16,7 \mathrm{~A}$ & $8,5 \mathrm{E}$ \\
\hline 120 (L3) & $7060,0 \mathrm{D}$ & $6263,9 \mathrm{D}$ & 0,97 & $0,043 \mathrm{ABC}$ & $16,2 \mathrm{~A}$ & $14,0 \mathrm{D}$ \\
\hline 240 (L4) & $10548,2 \mathrm{C}$ & $9554,7 \mathrm{C}$ & 0,97 & $0,046 \mathrm{AB}$ & $15,0 \mathrm{~A}$ & $18,9 \mathrm{C}$ \\
\hline 360 (L5) & $13683,4 \mathrm{~B}$ & $12303,0 \mathrm{~B}$ & 0,98 & $0,047 \mathrm{~A}$ & $15,0 \mathrm{~A}$ & $22,3 \mathrm{~B}$ \\
\hline 480 (L6) & $16939,0 \mathrm{~A}$ & $15436,2 \mathrm{~A}$ & 0,98 & $0,041 \mathrm{C}$ & $17,2 \mathrm{~A}$ & $25,3 \mathrm{~A}$ \\
\hline CV (\%) & 5,8 & 6,1 & & 3,9 & 5,9 & 5,5 \\
\hline
\end{tabular}

(1) C-degradado: quantidade de carbono liberado acumulado na forma de $\mathrm{CO}_{2}$ até os 135 dias; ${ }^{(2)} \mathrm{C}_{0}$ : carbono potencialmente mineralizável em 241 dias de incubação; ${ }^{(3)} \mathrm{R}^{2}$ : coeficiente de correlação do modelo de Stanford e Smith (1972); ${ }^{(4)} \mathrm{k} \mathrm{d}{ }^{-1}$ : taxa de decomposição por dia; ${ }^{(5)} \mathrm{T}_{1 / 2}$ : tempo de meia-vida. Médias seguidas pelas mesmas letras maiúsculas para as doses dentro de cada resíduo não diferem entre si. (Tukey a $5 \%$ ).

e anaeróbio, com degradação dos materiais facilmente decomponíveis, restando compostos orgânicos mais recalcitrantes à decomposição.

Para o composto orgânico, a estabilização das leituras iniciou aos 241 dias (Figura 3), tempo maior que para o lodo de esgoto, testado tanto no ensaio I como no ensaio II, evidenciando que a taxa de degradação do composto orgânico é menor do que aquela do lodo de esgoto devido à presença de substâncias húmicas de lenta degradação que são formadas durante o processo de compostagem (Amir et al., 2004). Contudo, para o composto orgânico, foi observada maior fração de degradação que o lodo de esgoto, quando se considera o mesmo tempo de realização do ensaio. A menor fração de degradação do composto de lodo de esgoto foi igual a 11,4\% (Quadro 2) para a dose C1, correspondente a $50 \%$ da necessidade de $\mathrm{N}$ da cultura da cana $(69,4$ $\mathrm{Mg} \mathrm{ha}^{-1}$ ), maior que a fração de 10,2\% obtida com a maior dose de lodo de esgoto aplicada $\left(42,4 \mathrm{Mg} \mathrm{ha}^{-1}\right)$.
Aos 241 dias, a maior fração de degradação do composto orgânico foi observada para a maior dose aplicada de $277,8 \mathrm{Mg} \mathrm{ha}^{-1}$ (Quadro 2) igual a $12,3 \%$. A maior fração de decomposição observada com a aplicação de composto poderia ser explicada pela melhoria nas propriedades químicas e físico-químicas do lodo de esgoto, quando submetido ao processo de compostagem, assim como pela redução de contaminantes disponíveis que poderiam interferir nos processos de decomposição microbiana (Fontaine et al., 2003).

A relação $\mathrm{C} / \mathrm{N}$ do lodo foi de 4:1, enquanto para o composto essa relação foi de 11:1, mais próxima no último caso à relação $\mathrm{C}: \mathrm{N}$ de solos (Quadro 1); portanto, a microbiota do solo estaria mais apta a decompor o composto orgânico, uma vez que estudos realizados mostram que os processos de mineralização são favorecidos quando a relação $\mathrm{C} / \mathrm{N}$ de materiais orgânicos incorporados ao solo devem ser no máximo de 20:1 (Derpsch et al., 1991; Lorenz e Lal, 2009). O processo é intermediado por meio de 


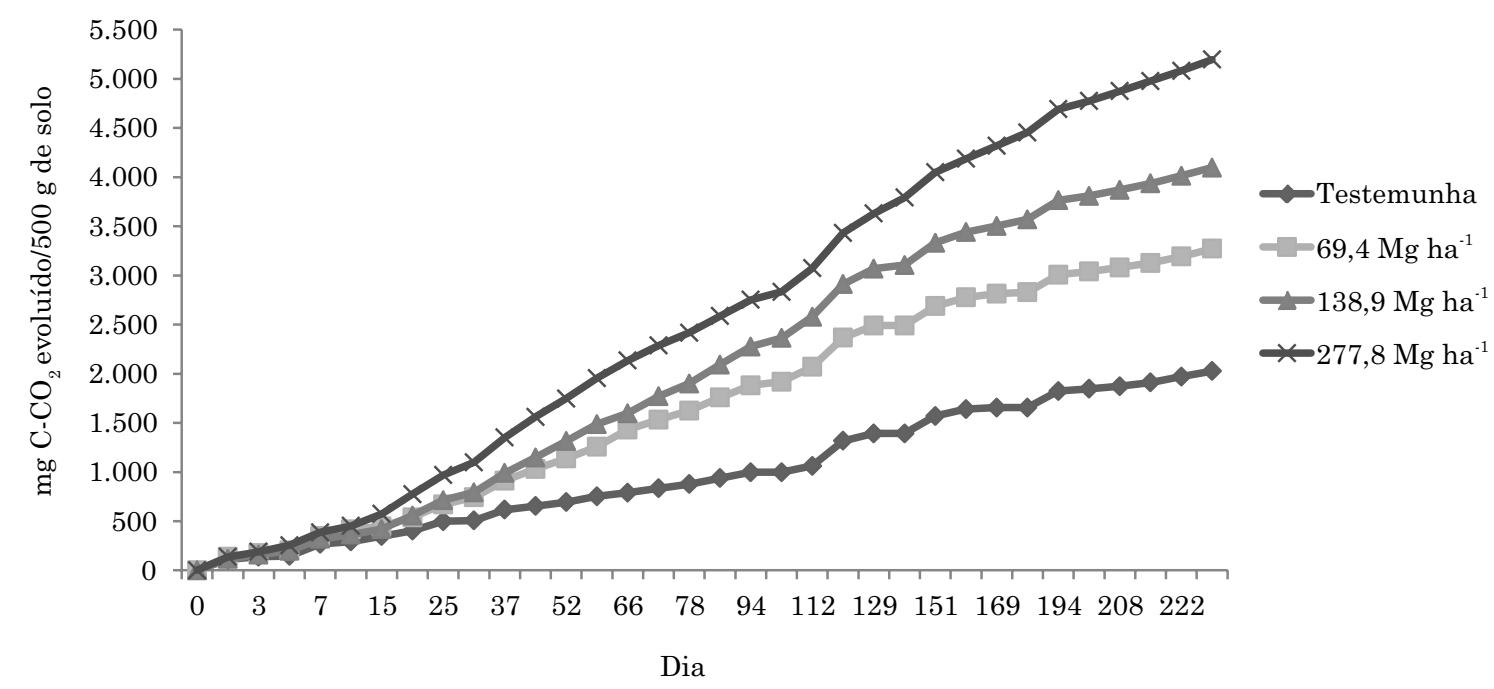

Figura 3. Evolução de C-CO $\mathrm{CO}_{2}$ em função das doses de composto de lodo de esgoto aplicadas em Nitolosso Háplico Álico, textura argilosa, coletado da camada de 0,00-0,20 m, de área localizada em Piracicaba, São Paulo.

microrganismos que promovem a redução da relação $\mathrm{C} / \mathrm{N}$ para valores em torno de 10:1 a 12:1, próximos aos valores encontrados na matéria orgânica nativa dos solos (Douglas et al., 1980). Também, verificase redução exata de $25 \%$ no teor de $\mathrm{Cu}, \mathrm{Zn}$, e $\mathrm{Mn}$ no composto orgânico quando comparado ao lodo (Quadro 1), em razão de fatores como a diluição desses na massa estruturante e, ou, maior retenção de contaminantes nos sítios de carga ativos do composto orgânico, representados pelos maiores valores de CTC/C observados no composto. Assim, a melhoria nas características químicas do composto e redução de contaminantes induziram maiores frações de decomposição do composto orgânico nesse solo argiloso.

A fração de degradação encontrada para a maior dose de composto de lodo de esgoto esteve dentro da faixa de 6 a $16 \%$ obtida em estudo realizado por Huang e Chen (2009), com doses de composto de lodo de esgoto em diferentes solos. Essa baixa fração de degradação pode indicar que o processo de compostagem foi completo, produzindo grande quantidade de materiais orgânicos estáveis de baixa e lenta degradação (D’Orazio et al., 2006).

As baixas frações de decomposição do material orgânico dos resíduos também se devem ao fato de o solo utilizado ter sido retirado da camada de 0,00-0,20 m de um Nitossolo Háplico Álico, textura argilosa. Sabe-se que a decomposição de resíduos orgânicos em solos argilosos sob condições tropicais é mais lenta em relação a solos mais arenosos, em razão da associação estável da matéria orgânica com minerais de carga variável, como caulinita, gibbsita, óxidos de $\mathrm{Fe}$, e a fração silte, que protegem a matéria orgânica do seu rápido decaimento (Bayer et al., 2002; Bertoncini et al., 2008).
Contudo, fica evidente, neste estudo, que a fração de degradação desse tipo de material orgânico em solo argiloso sob condição tropical está abaixo daquela estabelecida pela legislação do Estado de São Paulo, de 30 \% (Cetesb, 1999). Assim, para que o uso agrícola desse resíduo se torne realidade, é necessário realizar mais estudos que comprovem que frações de decomposição do C-total abaixo de $30 \%$ nem sempre representam prejuízos agrícolas ou ambientais ao sistema solo-água-planta, mas representam a presença de substâncias orgânicas de degradação mais lenta, que podem ser evidenciadas pelas substâncias húmicas, no caso do composto orgânico, e pela presença de compostos orgânicos de mais difícil degradação, no lodo de esgoto, resultantes de processo de tratamentos aeróbio e anaeróbio de esgotos domésticos (Andrade et al., 2006a; Boeira e Ligo, 2007), que contribuiriam com o aporte de matéria orgânica, tão almejado nesses tipos de solos.

Ajustando os resultados obtidos no ensaio de biodegradação de $\mathrm{C}$ à equação de cinética química de primeira ordem, observou-se que os coeficientes de correlação de todos os resultados, tanto para o ensaio I quanto para o ensaio II, estiveram em torno de 0,92 e 0,99 (Quadros 2 e 3), indicando bom ajuste dos dados a esse modelo matemático (Saviozzi et al., 1997; Reis e Rodella, 2002; Teles et al., 2009).

Por meio do ajuste dos resultados a essa equação, é possível determinar os valores de $\mathrm{C}$ degradado acumulado na forma de $\mathrm{CO}_{2}(C$-degradado $)$ durante o tempo de realização dos ensaios, de 241 dias para o ensaio I e 135 dias para o ensaio II. No ensaio I, observou-se que a dose L2 (200\% da necessidade de $\mathrm{N}$ da cana-de-açúcar) foi a que apresentou maior valor de $C$-degradado, seguido pela L1 que não 
diferiu do tratamento testemunha (Quadro 2). Para o composto orgânico notou-se diferença significativa entre todos os tratamentos, que apresentaram aumento da quantidade de $\mathrm{C}-\mathrm{CO}_{2}$ liberado com o aumento das doses de composto orgânico aplicado.

Quando se comparam as doses L1 e C2, que foram calculadas para fornecer $100 \%$ da necessidade em $\mathrm{N}$ para a cultura da cana-de-açúcar, observou-se que o valor de $C$-degradado foi maior para a dose C2. $\mathrm{O}$ mesmo comportamento foi notado para as doses de lodo de esgoto e composto de lodo de esgoto, que pretendiam fornecer $200 \%$ da necessidade em N para a cana-de-açúcar, a dose L2 e C3, confirmando os dados discutidos anteriormente.

Para os valores estimados da quantidade de C- $\mathrm{CO}_{2}$ potencialmente liberado pelos tratamentos $\left(C_{0}\right)$, não houve diferença entre as doses de lodo aplicadas e o tratamento testemunha. Diferentemente, os tratamentos com composto orgânico apresentaram diferença entre todos os tratamentos e aumento dos valores de $C_{0}$ com a elevação da dose de composto de lodo de esgoto, assim como observado para os valores de $C$-degradado. A dose $\mathrm{C} 3$, equivalente à maior dose de composto orgânico estudado, apresentou o maior valor de $C_{0}$.

Comparando as mesmas doses dos dois resíduos estudados, o composto orgânico apresentou maiores valores de $C_{0}$ para todas as doses, comportamento semelhante ao discutido anteriormente para os valores de $C$-degradado. Esses maiores valores da fração de degradação do material orgânico, $C$-degradado e $C_{0}$, observados para os tratamentos com composto orgânico quando comparados aos tratamentos com lodo de esgoto, provavelmente são em razão da adição de um material orgânico com melhores características químicas e físico-químicas (Fontaine et al., 2003) proporcionadas pelo processo de compostagem.

Tais resultados estão de acordo com o observado em estudo realizado com lodos de esgotos provenientes de diferentes sistemas de tratamento e um composto de lodo de esgoto com bagaço de cana-de-açúcar e resto de poda de árvore, conduzido por Andrade et al. (2006a), que encontraram frações de degradação variando de 5 a $22 \%$. Nesse mesmo trabalho, os autores encontraram baixa fração de degradação, igual a $7 \%$, para o lodo de esgoto proveniente da decantação de efluentes sanitário em lagoas, durante um ano, e posterior tratamento em lagoa aeróbia durante 120 dias, sistema de tratamento que proporciona maior remoção dos compostos facilmente decomponíveis.

A taxa de degradação $(k)$ dos tratamentos com lodo de esgoto se elevou com o aumento das doses aplicadas, diferindo entre si. Para o composto, observou-se maior taxa de degradação para a menor dose de composto orgânico, a dose $\mathrm{C} 1$, referente ao fornecimento de $50 \%$ da necessidade em $\mathrm{N}$ da cultura da cana-de-açúcar, seguido pelas doses C2, C3 e tratamento testemunha, que não diferiram entre si.

Quando as mesmas doses dos dois resíduos, L1 e C2 e L2 e C3, foram comparadas, observou-se que, para todas as doses, os valores da constante $k$ foram maiores para os tratamentos com lodo de esgoto, refletindo o menor tempo de ensaio requerido para esse tratamento para estabilizar as leituras no ensaio respirométrico.

O tempo de meia-vida $\left(T_{1 / 2}\right)$ apresentou comportamento inverso à constate $k$, ou seja, para os tratamentos com lodo de esgoto foram obtidos menores valores para as doses L1 e L2, quando comparados ao tratamento testemunha, como esperado para tais parâmetros. Para o tratamento com composto de lodo de esgoto, foram obtidos menores valores para a dose $\mathrm{C} 1$, seguido pelas doses C2 e C3 e tratamento testemunha, que não diferiram entre si.

Ao se compararem as mesmas doses de cada resíduo, percebeu-se que para todas as doses o composto apresentou maior tempo de meia-vida, provavelmente por causa da maior quantidade de compostos orgânicos mais estáveis, formados durante o processo de compostagem (D’Orazio, 2006; Bertoncini et al., 2008). Um ensaio de biodegradação de $\mathrm{C}$ conduzido por 70 dias foi ajustado por Andrade et al. (2006a), os quais também encontraram maiores valores para meia-vida de um composto de lodo de esgoto, quando comparado ao lodo de esgoto utilizado no processo de compostagem, com valores equivalentes a 33 dias para o composto orgânico e 11 dias para o lodo de esgoto.

Para o ensaio II, realizado somente com o lodo de esgoto, utilizando doses 5, 10, 15 e 20 vezes maiores que a dose que forneceria $100 \%$ da necessidade em $\mathrm{N}$ da cultura de cana-de-açúcar, observou-se que os valores dos parâmetros $C$-degradado, $C_{0}$, $e$ fração de degradação diferiram significativamente e evidenciaram-se crescentes com o aumento da dose de lodo de esgoto aplicada ao solo.

Em ensaio realizado com amostras de solo coletadas de área que recebeu sucessivas aplicações de lodo de esgoto, Andrade et al. (2013) também encontraram aumento do C- $\mathrm{CO}_{2}$ emanado com a elevação das doses de lodo de esgoto aplicadas no longo dos ciclos de cultivo, embora tenham observado redução nos valores da fração de degradação com o aumento das doses de lodo, o que foi atribuído pelos autores ao maior aporte de material orgânico nos tratamentos que receberam maiores doses de lodo de esgoto, ocasionando redução da eficiência da atividade microbiana em degradar o material. Frações de degradação crescentes com o aumento das doses de lodo de esgoto foram encontradas por Roig et al. (2012), em área com sucessivas aplicações de lodo de esgoto, 
elevando a dose acumulada no solo, tendência semelhante ao obtido por este estudo.

Neste estudo a taxa de degradação foi maior para a dose L5, seguido pelas doses L3, L4 e tratamento testemunha, e a maior dose de lodo de esgoto utilizada (L6) proporcionou redução no valor da constante $k$ (Quadro 3), indicando redução da taxa de degradação do resíduo, que pode ter ocorrido pelo acúmulo de material orgânico adicionado ao sistema, acima da capacidade de depuração do solo (Andrade et al., 2013), ou pela inibição da atividade dos microrganismos pela presença de poluentes/contaminantes em maior quantidade na dose de $480 \mathrm{Mg} \mathrm{ha}^{-1}$ de lodo de esgoto.

Os valores do tempo de meia-vida $\left(T_{1 / 2}\right)$ no ensaio II não diferiram entre si para os tratamentos testados, evidenciando a rápida decomposição do lodo, quando doses não agronômicas do resíduo são adicionadas ao solo (Quadro 3).

\section{CONCLUSÕES}

As frações de degradação do composto de lodo de esgoto foram maiores que as observadas para o lodo de esgoto, para as mesmas doses agrícolas estudadas, refletindo a melhoria da caracterização do resíduo proporcionada pelo processo de compostagem.

O composto de lodo de esgoto apresentou menor taxa de degradação e maior tempo de meia-vida, quando comparado ao lodo de esgoto, refletindo a natureza húmica do material compostado.

Somente a maior dose de lodo de esgoto aplicada ao solo, 20 vezes maior que a dose agrícola recomendada, esteve próxima do valor sugerido pela legislação paulista para fração de degradação de resíduos orgânicos em solos, indicando necessidade de atualizar a legislação para resíduos provenientes de tratamentos como a digestão anaeróbia e compostagem, utilizados em solos argilosos.

\section{AGRADECIMENTOS}

Ao CNPq, pelo financiamento da pesquisa (Processo $\mathrm{N}^{\circ}$ 575025/2008-5). À CAPES, pelo financiamento da bolsa de mestrado. À Sabesp/Franca, pelo apoio logístico ao processo de compostagem.

\section{REFERÊNCIAS}

Aita C. Dinâmica do nitrogênio no solo durante a decomposição de plantas de cobertura: efeito sobre a disponibilidade de nitrogênio para a cultura em sucessão. In: Fries MR, Dalmolin RSD, editores.
Atualização em recomendação de adubação e calagem: ênfase em plantio direto. Santa Maria: Pallotti; 1997. p.76-111.

Amir S, Hafidi M, Merlina G, Revel JC. Structural characterization of fulvic acids during composting of sewage sludge. Proc Biochem. 2005;40:1693-700.

Andrade CA, Oliveira C, Cerri CC. Cinética de degradação da matéria orgânica de biossólidos após aplicação no solo e relação com a composição química inicial. Bragantia. 2006a;65:659-68.

Andrade CA, Silva LFM, Pires AMM, Coscione AR. Mineralização do carbono e do nitrogênio no solo após sucessivas aplicações de lodo de esgoto. Pesq Agropec Bras. 2013;45:536-44.

Andrade JC, Abreu MF. Análise química de resíduos sólidos para monitoramento e estudos agroambientais. Campinas: Instituto Agronômico; 2006.

Andreoli CV. Alternativas de uso de resíduos do saneamento. Rio de Janeiro: ABES; 2006.

Bayer C, Mielniczuk J, Martin-Neto L, Ernani PR. Stocks and humification degree of organic matter fractions as affected by no-tillage on subtropical soil. Plant Soil. 2002;238:133-40.

Bertoncini EI, D'orazio V, Senesi N, Mattiazzo ME. Effects of sewage sludge amendment on the properties of two Brazilians Oxisols and their humic acids. Biores Technol. 2008;99:4972-9.

Bertoncini EI, Mattiazzo ME, Rossetto R. Sugarcane yield and heavy metal availability in two biosolid-amended Oxisols. J Plant Nutr. 2004;27:1243-60.

Boeira RC, Ligo MAV. Decomposição de lodos de esgoto em Latossolos. R Bras Agroecol. 2007;2:208-11.

Bovi MLA, Godoy-Júnior G, Costa EADC, Berton RS, Spiering SH, Vega FVA, Cembranelli MAR, Maldonado CA. Lodo de esgoto e produção de palmito em pupunheira. R Bras Ci Solo. 2007;31:153-66.

Brasil. Conselho Nacional do Meio Ambiente - Conama. Resolução $\mathrm{N}^{\circ} 375$, de 29 de ago. 2006. Define critérios e procedimentos, para uso agrícola de lodos de esgoto gerados em estações de tratamento de esgoto sanitário e seus produtos derivados, e dá outras providências. Diário Oficial da União, Brasília, DF, 30 de ago. 2006. Seção 1. p.141-6, 2006a.

Brasil. Conselho Nacional do Meio Ambiente - Conama. Resolução $\mathrm{n}^{\circ} 380$, de 31 de out. 2006. Define critérios e procedimentos, para uso agrícola de lodos de esgoto gerados em estações de tratamento e dá outras providências. Diário Oficial da União, Brasília, DF, 7 de nov. 2006. seção 1. p.59. 2006b.

Brasil. Lei 12.305. Institui a Política Nacional de Resíduos Sólidos; altera a lei 9.605 , de 12 de fev. 1998 e dá outras providências. Diário Oficial da União, Brasília, DF, 3 de ago. 2010. p.2. 2010.

Companhia de Tecnologia de Saneamento Ambiental - Cetesb. Norma P4.230 - Critérios para aplicação de biossólidos em áreas agrícolas: critérios para projeto e operação. São Paulo: Cetesb; 1999. (Manual técnico).

Corrêa RS, White RE, Weatherley AJ. Effect of compost treatment of sewage sludge on nitrogen behavior in two soils. Waste Manage. 2006;26:614-9.

Derpsch R, Roth CH, Sidiras N, Kopke U, Krause R, Blanken J. Controle da erosão no Paraná, Brasil: sistemas de cobertura do solo, plantio direto e preparo conservacionista do solo. Eschborn: GTZ; 1991. 
D’orazio V, Traversa A, Stefanutti R, Bertoncini EI, Senesi N. Humic Acid-like substances in composts produced in tropical regions. Proceedings of the $13^{\text {th }}$ Meeting of the International Humic Substances Society [CD-ROM]; 2006; Karlsruhe. Karlsruhe: Universität Karlsruhe; 2006.

Douglas CL, Allmaras RR, Rasmussen PE, Ramig RE, Roager NC. Wheat straw composition and placement effects on decomposition in dryland agriculture of the Pacific Northwest. Soil Sci Soc Am J. 1980;44:833-7.

Firmino MC, Farias MSS, Medeiros SS, Guerra HOC, Guimarães JP. Características químicas do solo influenciadas pela adição de água residuária tratada sob cultivo do pinhão manso. Agropec Cient Semiárido. 2015;11:32-7.

Fontaine S, Mariotti A, Abbadie L. The priming effect of organic matter: a question of microbial competition? Soil Biol Biochem. 2003;35:837-43.

Franco A, Abreu-Junior CH, Perecin D, Oliveira FC, Granja, ACR, Braga VS. Sewage sludges as nitrogen and phosphorus source for cane-plant and first ratoon crops. R Bras Ci Solo. 2010;34:553-61.

Guedes MC, Andrade CA, Poggiani F, Mattiazzo ME. Propriedades químicas do solo e nutrição do eucalipto em função da aplicação de lodo de esgoto. R Bras Ci Solo. 2006;30:267-280.

Hernandez T, Garcia C, Lax A. Transformation of carbon and nitrogen in a calciorthid soil amended with a range of organic residues. Plant Soil. 1988;105:205-11.

Hsieh YP, Lowell AD, Motto HM. Modeling sewage sludge decomposition in soil: I. Organic carbon transformation. J Environ Qual. 1981;10:54-64.

Hua L, Wu W, Liu Y, Mcbride MB, Chen Y. Reduction of nitrogen loss and $\mathrm{Cu}$ and $\mathrm{Zn}$ mobility during sludge composting with bamboo charcoal amendment. Environ Sci Pollut Res Int. 2009;16:1-9.

Huang CC, Chen ZS. Carbon and nitrogen mineralization of sewage sludge compost in soils with a different initial $\mathrm{pH}$. Soil Sci Plant Nutr. 2009;55:715-24.

Khalil AI, Hassouna MS, El-Ashqar HMA, Fawzi M. Changes in physical, chemical, and microbial parameters during the composting of municipal sewage sludge. World J Microbiol Biotecnol. 2011;27:2359-69.

Kiehl EJ. Fertilizantes orgânicos. Piracicaba: Agronômica Ceres; 1985.
Latham JL. Cinética elementar de reação. São Paulo: Edgard Blücher; 1974.

Lorenz K, Lal R. Biogeochemical C and N cycles in urban soils. Environ Int. 2009;35:1-8.

Medeiros SS. Crescimento e produção do pinhão manso sob adubação fosfatada e irrigação com água residuária [dissertação]. Campina Grande: Universidade Federal de Campina Grande; 2012.

Melo WJ, Aguiar PS, Melo GM, Melo VP. Nickel in a tropical soil treated with sewage sludge and cropped with maize in a long-term field study. Soil Biol Biochem. 2007;39:1341-7.

Motaghian A, Bahmanyar MA. Effects of organic amendments application in grain yield, leaf, chlorophyll content and some morphological characteristics in soybean cultivars. J Plant Nutr. 2010;33:485-95.

Pereira Neto JT. Conceitos modernos de compostagem. R Eng Sanit. 1989;28:104-9.

Reis TC, Rodella AA. Cinética de degradação da matéria orgânica e variação do $\mathrm{pH}$ do solo sob diferentes temperaturas. $\mathrm{R}$ Bras $\mathrm{Ci}$ Solo. 2002;26:610-26.

Rodella AA, Saboya LV. Calibration for condutimetric determination of carbon dioxide. Soil Biol Biochem. 1999;31:2059-60.

Roig N, Sierra J, Martí E, Nadal M, Schuhahcer M, Domingo JL. Long-term amendment of Spanish soils with sewage sludge: Effects on soil functioning. Agric Ecosyst Environ. 2012;158:41-8.

Saviozzi A, Levi-Minizi R, Riffaldi R, Vanni G. Role of chemical constituents of wheat straw and pig slurry on their decomposition in soil. Biol Fertil Soils. 1997;25:401-6.

Stanford F, Smith SJ. Nitrogen mineralization potentials of soil. Soil Sci Soc Am J. 1972;36:465-72.

Teles CR, Munaro CJ, Cassini STA. Modelagem da decomposição aeróbia de lodo de esgoto em solos com diferentes texturas. R Bras Eng Agríc Amb. 2009;13:197-203.

Victoria RL, Piccolo MC, Vargas AAT. O ciclo do nitrogênio. In: Cardoso EJBN, Tsai, SM, Neves MCP, editores. Microbiologia do Solo. Campinas: Sociedade Brasileira de Ciência do Solo; 1992. p.105-20.

Wang X, Chen T, Ge Y, Jia Y. Studies on land application of sewage sludge and its limiting factors. J Hazard Mater. 2008;160:554-8. 